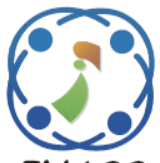

\title{
ECTTM - Energy Conserving Trustworthy Topology Management Mechanism for Wireless Sensor Networks
}

\author{
Theagarajan Senthil $^{1 *} \quad$ Balasubramanian Kannapiran $^{2}$ \\ ${ }^{1}$ Kalasalingam University, Krishnankoil, India \\ * Corresponding author's Email: t.senthilklu@gmail.com
}

\begin{abstract}
Energy conservation is the major concern of Wireless Sensor Networks, as the energy is the determining factor of the network lifetime. The reason is that the energy level of sensors has a great impact on the network lifetime. Several mechanisms have been proposed to minimize the energy consumption of nodes. In this paper, a cluster based, trustworthy topology management mechanism is presented and is proved to be energy efficient. The entire work relies on three important phases and they are cluster formation, chief node selection, trust degree computation and node's state control. The cluster is formed by taking the location coordinates into account. This is followed by the selection of chief node. The chief node is responsible for computing the trust degree and to control the state of the constituent nodes. As limited count of nodes is involved in the process, the lifetime of the network is considerably improved. Finally, the proposed work is tested for its efficacy and the experimental results are satisfactory with respect to network lifetime.
\end{abstract}

Keywords: Energy efficiency, Network lifetime, Wireless sensor networks, Trust.

\section{Introduction}

Wireless Sensor Networks (WSN) are composed of numerous sensor nodes, which are geographically scattered for achieving certain tasks. The nodes communicate with each other and submit the sensed data to the Base Station (BS) periodically. As the applicability of WSN is broader, it is hard to predict the nature of tasks assigned to the sensors. The sensor's task can range from simpler to complex. For instance, the simpler task can be data collection and the complex task can be some manipulation with the collected data. Thus, the network can serve its purpose only when the lifetime network is reasonable.

The sensor nodes are constrained in terms of energy, memory and computational capability. Hence, it is necessary to utilize the available resources in an efficient manner. Energy efficiency is the major issue in WSN, as it is directly proportional to the lifetime of the network. There are several ways to achieve energy efficiency and a vast literature already exists for the same. Some of the popular ways to achieve energy efficiency are radio optimization, data reduction, sleep/wakeup schemes, energy efficient routing and battery repletion [1].

This article aims to conserve energy by means of sleep/wakeup mechanism. The objective of sleep/wakeup mechanism is to save the energy of sensors, by setting the radio into sleep mode, whenever necessary. The sleep/wakeup mechanism can be achieved by duty cycling scheme, passive wakeup radios and topology management techniques.

Duty cycling schemes switch between the idle listening, sleep states, with respect to the network activity. This mechanism is energy efficient but sleep latency is experienced. Besides this, the duty cycling techniques consume more energy for needless wakeups. Passive wakeup scheduling requires a passive entity to perform wakeup. This practise consumes more energy. Topology management mechanism manages the network connectivity by limiting the count of functioning 
nodes. Thus, the minimal count of functioning nodes is set with respect to the nature of the application.

The main intention of this paper is to conserve as much energy as possible, and thereby increasing the lifetime of the network. The aforementioned objective is attained by means of Energy Conserving Clustered Trustworthy Topology Management (ECCTTC) mechanism. The nodes are clustered with respect to the geographical coordinates and the cluster head is selected by considering the trust metrics. The proposed scheme conserves more energy by fixing the minimal count of functioning nodes. Thus, the state of the remaining nodes is set to sleep. This idea ends up in energy preservation, which leads to network lifetime maximization. Some of the highlights of this paper are presented below.

- The sensors are clustered based on the geographical location and thus the node's mobility has nothing to play with the clustering operation. Hence, the WSN can either be mobile or immobile.

- The cluster head is selected by considering the energy consumption pattern of nodes.

- The cluster head monitors the behaviour of cluster member nodes and controls the count of functioning nodes. This improves the network security and the consumption of energy is considerably reduced.

- As the proposed algorithm is based on clustering approach, the scalability and robustness are rendered along with minimized energy consumption and latency.

- The cluster head is attentive towards the misbehaviour and so it computes the trust degree for all of its constituent nodes. The cluster head node blocks the member node when the trust score is very low.

- The proposed algorithm involves no memory overhead, as the nodes need not to maintain any tables. The cluster head alone maintains two tables to store recent routes and trust degree of member nodes.

The rest of the paper is organized as follows. Section 2 deals with the related review of literature. The proposed methodology along with the preliminaries is presented in section 3. Section 4 evaluates the performance of the proposed approach and the experimental results are presented. In the end, the concluding remarks are presented in section 5.

\section{Review of literature}

This section is dedicated to present the related works with respect to topology management techniques.

A topology management mechanism controls the participating nodes of WSN by altering the transmission range or by choosing nodes to achieve tasks [2]. The topology management schemes can be classified into homogenous and heterogenous methods. The homogenous schemes employ nodes with the same transmission range. On the other hand, the heterogenous schemes differ in terms of transmission range [3].

There are many ways to control the topology of the network. Some of them are transmit power alteration, sleep cycle scheduling, location information are clustering techniques [4]. The concept of clustering controls the topology by balancing the load of nodes and thereby increasing the network lifetime [5].

The Dead-end Free Topology Maintenance (DFTM) protocol is proposed in [6], which employs limited functioning nodes for packet forwarding capability. This protocol utilizes geographical location information, while forwarding packets and this leads to energy conservation. However, this work suffers from computational overhead and location information is the main ingredient of this algorithm. A multi-objective cluster based routing protocol is presented in [7], which tackles the issues of routing and area coverage. This protocol conserves maximum energy and attains better area coverage. Cluster based approaches are proved to be easily manageable and scalable. The main drawback of this technique is the coverage and connectivity issues.

Most of the cluster based protocols utilize the Connected Dominating Set (CDS) approach. Numerous topology control mechanisms make use of CDS approach for conserving energy. Dominating Set (DS) is the set which consists of several set of nodes and the DS make sure that at least node from a subset has the direct connection with the node present in other subsets [8]. When the nodes of dominating set construct a connected graph, then the set is called as CDS. In the literature, there are three popular topology control algorithms based on clustering approach.

In [9], a topology control algorithm namely Power Aware Connected Dominating Set (PACDS), which is based on CDS is presented. This algorithm constructs the CDS by means of marking process. The major goal of this algorithm is to construct smaller CDS and to improve the network lifetime. Though this algorithm aims to improve the network lifetime, complex computations consume more 
energy, which results in the performance degradation. An Energy Efficient Distributed Connecting Dominating Sets (ECDS) is presented in [10] for topology control. The ECDS algorithm relies on two important phases. The initial phase is responsible for the computation of Maximal Independent Set (MIS). MIS is the Independent Set (IS), which is not a subset of other IS [11]. The initial phase aims to detect the MIS nodes by colouring technique and the second phase connects the MIS. This algorithm involves technical complexity and cannot be achieved easily.

A dynamic algorithm for topology control namely Topology Management by Priority Ordering (TMPO) is presented in [12]. This algorithm works dynamically by taking energy and mobility rate of nodes into account, while forming the backbone. This algorithm preserves more energy and involves minimal overhead. The topology is formed by taking the energy and mobility rates into account and this algorithm does not focus to improve the quality of service.

Motivated by these works, this article aims to present an energy efficient algorithm for topology control in WSN. The proposed algorithm roots on clustering concept, which conserves the energy at its best. The cluster head selection is achieved by the energy consumption pattern. Besides this, the count of functioning nodes is controlled and thus, the energy is conserved to the maximum. The proposed algorithm is presented in the forthcoming section.

\section{Proposed approach}

This section aims to demonstrate the overall flow and the working principle of the proposed work.

\subsection{Outline of the work}

The main intention of this paper is to propose a cluster based trustworthy topology management mechanism, which conserves energy to the maximum and thereby enhances the network lifetime. The nodes are clustered on the basis of the geographical location of the nodes. The area of the network is partitioned into several square shaped grids. As the locational coordinates play a vital role in the process of clustering operation, the mobility of the nodes doesn't have any impact over the clustering process. The overall working nature of the proposed algorithm can be organized into

- Network area splitting phase,

- Clustering phase,

- Nodes' state control phase.
Initially, the area of the network is divided into same sized, square shaped grids. The nodes present within a grid form the cluster and thus the count of cluster member nodes varies from grid to grid. The next objective of this work is to select a chief node to govern and control the member nodes effectively. Hence, the chief node must be selected with intense care. The chief node manages the member nodes, such that the misbehaviour of nodes can be detected easily. The chief node monitors its member nodes continuously, such that the malicious nodes can be blocked. Besides this, the concept of clustering contributes several merits such as scalability, effective maintenance and energy conservation. The chief node decides the state of the member nodes. The member nodes can either be in functioning or sleeping state. The count of functioning nodes in every grid is limited, such that the remaining nodes are in sleep state. Whenever the energy of the functioning nodes fall below the energy threshold or when the time slot expires, the functioning node is recycled. The maximum count of functioning nodes per grid is set as two. Thus, the energy is conserved at its best and the lifetime of the network is enhanced further. All these phases are elaborated in the following sections and the assumptions of the work are presented below.

- A single mobile base station which is equipped with high energy backup is included in the network.

- The sensors present in the network are stationary and static.

- All the nodes participating in the network is aware of its' locational coordinates and they are clustered with respect to the locational coordinates.

- The nodes of the network may play the role of the cluster head or the member node.

- This paper denotes cluster head as the chief node and the cluster member as constituent node.

- A constituent node can send the packet to the chief node directly.

- The chief node sends the data to the BS via other chief nodes.

- As the nodes involved in this work are static, the nodes are uniformly distributed over all the grids.

- The minimum count of functioning nodes per grid is fixed as two. 


\subsection{Network area splitting phase}

The main target of this phase is to partition the overall network area into equal sized grids. The noteworthy point of this technique is that the nodes residing in a particular geographic location forms a cluster. Another important point is that the count of nodes per grid is not predefined. A grid can accommodate any number of nodes and the minimum count of functioning nodes per grid is two. Thus, certain clusters may be overcrowded and other clusters may possess limited nodes. In order to handle this issue, this work uniformly distributes the nodes over the grids. Each and every grid of this work is loaded with fixed count of nodes. As the clustering process depends on the locational coordinates, the mobility of the nodes does not affect the cluster process. The nodes can be highly mobile or immobile. However, this paper employs the immobile nodes and the Base Station (BS) alone is mobile. The network area is equally divided into several grids which are of equal size. The sensors are evenly scattered in all the grids.

\subsection{Cluster construction}

As the cluster of nodes is established with respect to geographical coordinates, there is no complexity involved in the formation of cluster. However, selecting the perfect chief node is a challenge. Additionally, it is not a good idea to retain the same node as cluster head. The reason for this is that the energy of a single node will get drained completely. Thus, it is necessary to recycle the chief node based on some criteria. This work recycles the chief node by setting the time or energy threshold as the recycling criteria. There are two sub-phases involved in this cluster construction phase and they are chief node selection and recycling phases. The sub-phases are described in the following subsections.

\subsubsection{Chief node selection process}

When this work is concerned, the cluster of nodes is already constructed by taking the location coordinates into consideration. The chief node must be selected on the basis of a suitable criterion. Chief node selection is a crucial task, because the chief node should be able to manage all the member nodes. This work selects the chief node by considering the energy consumption pattern of the nodes. The energy consumption pattern can be framed only when, the behaviour of the nodes is observed for a certain period. Thus, initially the behavioural pattern of all the nodes is observed for a time interval $t_{i}$.

All the nodes present in the network circulates an Strt_msg, which contains the unique identifier of the node and the available energy avl_erg. The Strt_msg is broadcasted within the grid. Thus, many nodes receive Strt_msg from the nearby nodes. When a node receives Strt_msg, the distance of the message sent node is computed by the signal power. The energy consumption pattern of the node is calculated by

$$
\operatorname{erg}_{\text {crate }}(i)=\frac{\text { cons }_{\text {erg }(i)}}{\text { avl_erg }(i)}
$$

$\operatorname{erg}_{\text {crate }}(i)$ denotes the energy consumption rate of the $i^{\text {th }}$ node, cons $_{\text {erg(i) }}$ is the consumed energy of the $i^{\text {th }}$ node and avl_erg(i) is the currently available energy. The time it takes for broadcasting the $c n_{m s}$, which constitutes the unique identifier of the node is computed by the following formula.

$$
\text { btime }_{t}(i)=\operatorname{erg}_{\text {crate }}(i) \cdot \text { rand }
$$

rand is the random number which ranges between 0.5 and 1 . The purpose of the rand is to prevent the $c n_{m s g}$ being sent at the same time and thereby minimizing the collision rate. Besides this, the energy consumption is also reduced considerably. The waiting time of the nodes to receive $c n_{m s g}$ is lesser for the minimal energy consuming nodes. This makes sense that the chance of receiving $c n_{m s g}$ by the minimal energy consuming nodes is low, because of its short waiting time. The basic idea behind this point is that the energy conserving node best suitable to act as the chief node.

All the nodes of the network wait for the $c n_{m s}$

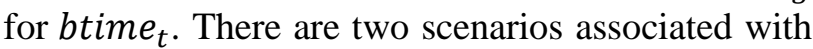
this. The first scenario is with the nodes that receive $c n_{m s g}$ and the second scenario indicates the nodes that do not receive $c n_{m s g}$. On receiving the $c n_{m s g}$, the node computes the distance between the $c n_{m s g}$ sent node and itself. In case, if the node does not receive $c n_{m s g}$, then that particular node shows interest to play the role of the chief node. This way of circulating $c n_{m s g}$ does not involve any collision, owing to the message circulation within specific grids and the employment of rand function. The algorithm for chief node selection is given below. 


\section{ECTTC - Chief node selection algorithm}

Input: Network grids with nodes,

Output: Chief node selection

Begin

//Activity Observation phase

Broadcast Strt_msg within specific grids;

Until ( $t 1$ expiry) do

Receive Strt_msg;

Compute erg $_{\text {crate }}$ \&btime $_{t}$;

Calculate the distance and save in the local table;

End;

// Chief node selection

If (tp2 is not expired) do

Wait till btime for $^{\mathrm{C}} n_{m s}$;

If $\left(\mathrm{Cn}_{\text {msg }}\right.$ received $)$

Set node_status=constituent node;

If (no $C n_{m s g}$ received)

Broadcast $C n_{m s g}$ within the specific grid;

Set node_status $=$ chf_node;

End;

//Chief node recycling

Input: Chief nodes

Start

Check if (available energy $\leq$ energy threshold $\|$ time slot expiry)

Obtain BS consent;

Recycle the node;

End;

End;

The above presented algorithm intends to select the most suitable node as the chief node and it is not recommendable to maintain the same node as chief node for a long time. Hence, it is necessary to recycle the chief node based on some criteria. The proposed algorithm recycles the chief node by setting two different decisive factors and they are the energy level of the chief node and the expiration of the time slot. The energy threshold is fixed as 0.6 and the time slot is set as two minutes. Whenever the energy threshold goes below the energy threshold, a caution alarm is generated by the chief node and a new chief node is selected by the BS. In the second case, for every time slot the available energy level of the chief node is checked and recycled by the $\mathrm{BS}$, if necessary.

This idea preserves more energy and the communication overhead is effectively managed. Besides this, the energy conserving node is selected as the chief node. The main purpose of the chief node is to manage its constituent nodes and thus the performance of the chief node must be superior to the constituent nodes. This is the reason for why keen attention is rendered towards chief node selection. The chief node is in charge of computing the trust degree of its constituent nodes and to decide the state of the constituent nodes. The functionality of the chief node is presented in the next section.

\subsubsection{Functionality of the chief node}

The major functionality of the chief node is to compute the trust degree of its constituent nodes and to manage the state of the constituent nodes, so as to save as much energy as possible. The trust degree of the constituent nodes is computed by taking vital trust metrics such as Packet Forwarding Ratio (PFR) and the Energy Backup (EB) into account. This trust rate computation is done by employing Bayes' theorem [13], which is proposed by Rev. Thomas Bayes. The next subsections present the trust degree computation and the state management by the chief node.

\subsubsection{Trust degree computation}

After the successful selection of the chief node, the chief node is expected to perform its duties. The two major duties being allotted to the chief node are trust degree computation and the state management of constituent nodes. The trust degree computation is necessary, such that the behaviour of the nodes can be tracked and the malicious nodes can be figured out. The trust degree of the nodes is computed by taking two important trust metrics into account, which are PFR and EB of the nodes. PFR is considered because this metric shows the cooperation level of the node in forwarding the packets. The EB is taken into account, as it is the key requirement of any node to satisfy its allotted tasks. Besides this, if the EB is low then the node cannot fulfil the purpose for a long time. Considering the aforementioned statement, these two metrics are chosen and the trust degree is computed by the Bayes' theorem, as given below.

$$
P\left(t d \mid p f r, e_{b}\right)=\frac{P\left(p f r \mid t d, e_{b}\right) \times P\left(t d \mid e_{b}\right)}{P\left(p d r \mid e_{b}\right)}
$$

In Eq.(3), $P\left(t d \mid p f r, e_{b}\right)$ is the posterior probability. $P\left(p f r \mid t d, e_{b}\right)$ is the prior probability. $P\left(t d \mid e_{b}\right)$ is the likelihood and it gives the probability of trust degree for the energy backup and $P\left(p f r \mid e_{b}\right)$ is the normalizing factor.

The likelihood function of $P\left(p f r \mid t d, e_{b}\right)$ is $P(p f r \mid t d)$. The probability of trust degree is presented by the following equation.

$$
P\left(t d \mid p f r, e_{b}\right)=\frac{P(p f r \mid t d) \times P\left(t d \mid e_{b}\right)}{P\left(p f r \mid e_{b}\right)}
$$


Bayes' theorem computes the $P(p f r \mid t d)$ as follows.

$$
P(p f r \mid t d)=\frac{P(t d \mid p f r) \times P(p f r)}{P(t d)}
$$

Eq. (5) is applied in Eq. (4) and the resultant equation is presented below.

$$
P\left(t d \mid p f r, e_{b}\right)=\frac{P(t d \mid p f r) \times P\left(t d \mid e_{b}\right) \times P(p f r)}{P\left(p f r \mid e_{b}\right) \times P(t d)}
$$

The normalizing factor is eliminated and the resultant equation is given in Eq. (7).

$$
P\left(t d \mid p f r, e_{b}\right)=P(t d \mid p f r) \times P\left(t d \mid P\left(t d \mid e_{b}\right)\right.
$$

The maximum trust value that can be obtained by this bayes' theorem is 1 . If the node's in-ratio is equal to the out-ratio of packets and if the node is fully energized, then the trust rate is 1 . Trust degree may turn to 0 if the packet delivery ratio and the energy backup is not up to the level.

By this way, the trust degree of nodes is computed and stored in the chief node and the computed trust degree is reported to the BS also. The trust degree doesn't need to be computed often, as the nodes are immobile. Besides this, only limited count of nodes are set to be in active state. This reduces the computational and memory overhead further. Based on the computed trust degree, the functioning nodes are selected by the chief node. The functioning nodes selection procedure is presented in the following section.

\subsubsection{Node state management}

The state of the constituent nodes is decided by the chief node with respect to the trust degree of the constituent nodes. The maximum count of the functioning nodes is fixed as two. As soon as the trust degree is computed, the trust degree of all constituent nodes is stored in the chief node. The top ranking two nodes are selected as the functioning nodes for a time slot. The functioning nodes are recycled when the energy backup of the nodes goes below the threshold or when the time slot is expired. In case, if the time slot expires and the energy backup is still greater than the energy threshold, then the same node can be in functioning mode. In case, if the energy of both the functioning nodes is lesser than the energy threshold then the chief node computes the trust degree and selects the functioning nodes again. However, the functioning nodes of the first round are not selected for the second round. The different cases are presented below.

\section{Case 1: Recycle both functioning nodes}

The first case illustrates the scenario in which the energy of both the functioning nodes falls down below the energy threshold, within or after the expiry of the time slot. In this scenario, the chief node computes the trust degree of the constituent nodes and the top ranking nodes are selected as the functioning nodes.

\section{Case 2: Recycle single functioning node}

In this case, the energy of a single node drops below the energy threshold, out of two functioning nodes. The energy of the node can either be dropped within or after the expiry of the time slot. Hence, only one functioning node has to be selected. In this case, the chief node checks the local memory and selects the top ranked node as the functioning node.

\section{Case 3: Recycle no functioning node}

This case signifies the scenario in which the recycling procedure of functioning nodes is not required. This makes sense that the energy of the functioning nodes is pretty higher than the energy thresholds and thus the same functioning nodes proceed to operate for the next time slot too.

By this way, the functioning nodes are selected. It is observed that more energy is conserved as the functioning nodes are scheduled by the chief node, on the basis of the trust degree. As the functioning nodes are selected with respect to trust score, the entire process is secured. This is because, the nodes with malicious activity are blocked immediately and reported to the BS. The next section intends to analyse the performance of the proposed work.

\section{Experimental analysis}

The performance of the proposed ECTTM is evaluated against ECTMRA [14] with respect to energy consumption and network lifetime. Besides this, the performance of ECTTM is analysed by varying the functioning nodes and number of nodes per grid. The experimental area of the proposed work is set as 100 by 100 metre square and the each sensor grid is of size 10 by 10 metre square area. During network initialization, all the nodes are energized with 60 joules. The network lifespan is measured by differentiate the number of nodes from 300 to 2000 and the functioning nodes are changed as 2, 5 and 10 respectively. 


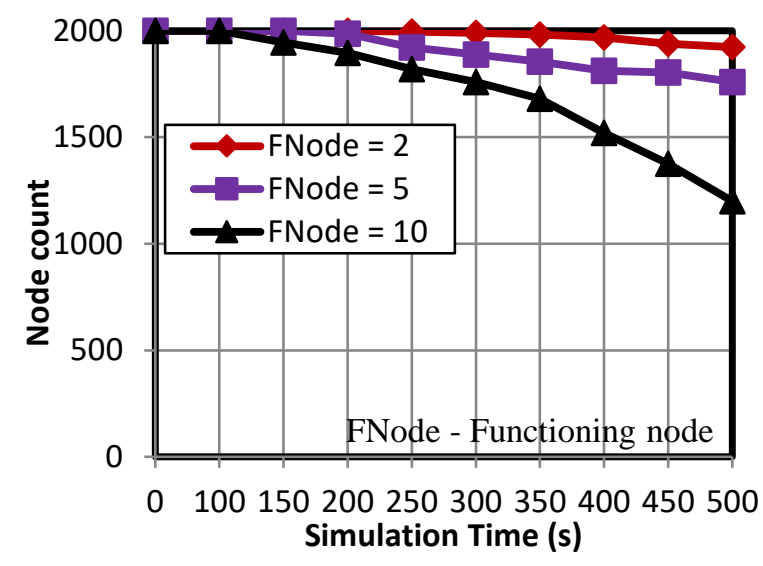

Figure.1 Network lifespan w.r.t functioning nodes

\subsection{Network lifespan by varying the functioning nodes}

The count of functioning nodes is differentiated, in order to study the impact of functioning nodes over the lifetime of the network. It is observed that the increased count of functioning nodes reduces the lifetime of the network. The experimental analysis is done with respect to simulation time against the total count of nodes. The experimental results are shown in the below given Fig.1.

The impact of the functioning node is checked by varying different counts of functioning nodes. It is obvious that the lesser number of functioning nodes consume minimal energy, which contributes to enhance the network lifetime. In order to prove the capability of the choice of Fnode, this work analyses the work by varying FNode count to 2,5 and 10. When the Fnode is 2, the lifetime of the nodes is better. On observing the experimental results, it is clear that the lifetime of the network decreases as the number of functioning nodes increases. The functioning nodes are denoted as FNode. When the count of functioning nodes is fixed as 10 , the lifetime of the network decreases sharply.

\subsection{Network lifetime by varying the nodes per grid}

The count of nodes being deployed is varied in order to check the lifetime of the network. The count of nodes per grid is varied between 5 and 20 and the lifetime of the network is analysed. On experimentation, it is observed that the number of nodes is increased with minimal functioning nodes, then the network lifetime is enhanced. On the other hand, when the count of functioning nodes is increased, then the network lifetime is affected. The experimental results are shown in Fig.2.

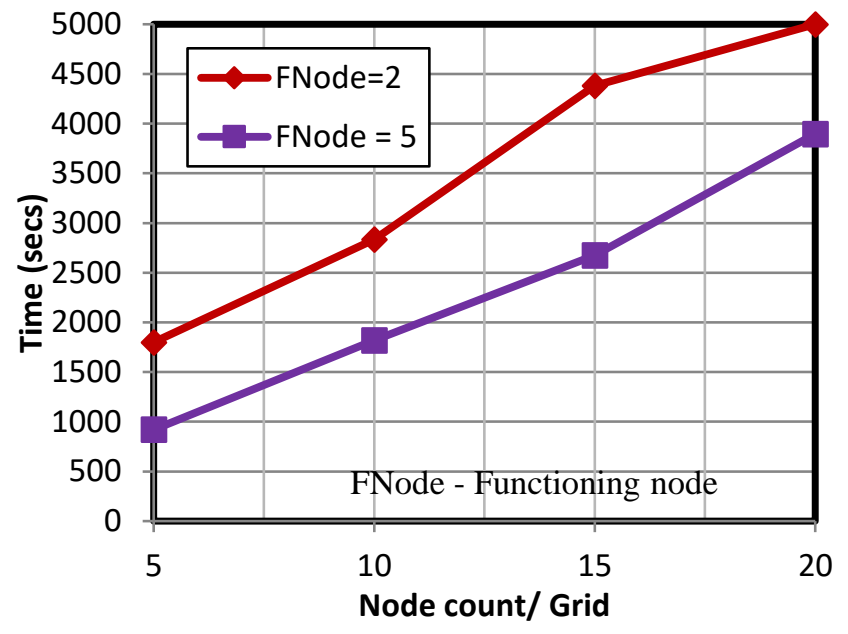

Figure.2 Network lifetime w.r.t node count per grid

This section attempts to prove the importance of fixing the count of nodes per grid and for each grid, the functional nodes are varied. In order to illustrate this, four different experimental procedures are carried out. Initially, each grid is loaded with five nodes and two nodes are set as operating nodes and the energy of the nodes are consumed quickly and the lifetime of the network is 1800 secs. On the other hand, when the number of functioning nodes is set as five, all the nodes in the grid are in work state. This leads to the network lifetime deterioration, which is only 920 seconds. Similarly, the experimentations are carried out for 10,15 and 20 nodes per grid and the functional nodes are varied as 2 and 5. When the functional nodes are set as 5, the performance of the network is better, however at the cost of energy consumption. When the number of nodes per grid is set as twenty with two functional nodes, the network lifetime is 5000 seconds. On the other hand, when the functional nodes are set as 5, the network lifetime suddenly falls to 3897 seconds.

In this analysis, the count of nodes per grid is varied and the network lifetime is measured. On analysis, it is observed that the network lifetime is improved when the node count per grid is increased and the functioning nodes are limited to 2 .

\subsection{Energy consumption}

The energy consumption analysis of ECTTM is done against ECTMRA. ECTMRA doesn't utilize any grids to deploy sensors and the nodes duty cycles are not maintained. Thus, the proposed ECTTM proves better performance than ECTMRA with respect to network lifetime. The results are presented in the following Fig.3. The reason for the better performance of ECTTM is that ECTMRA 


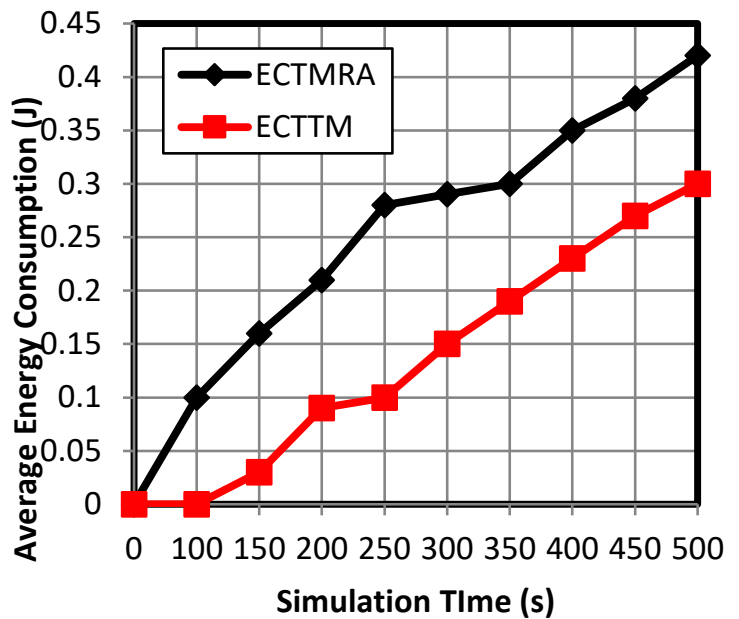

Figure.3 Energy consumption analysis

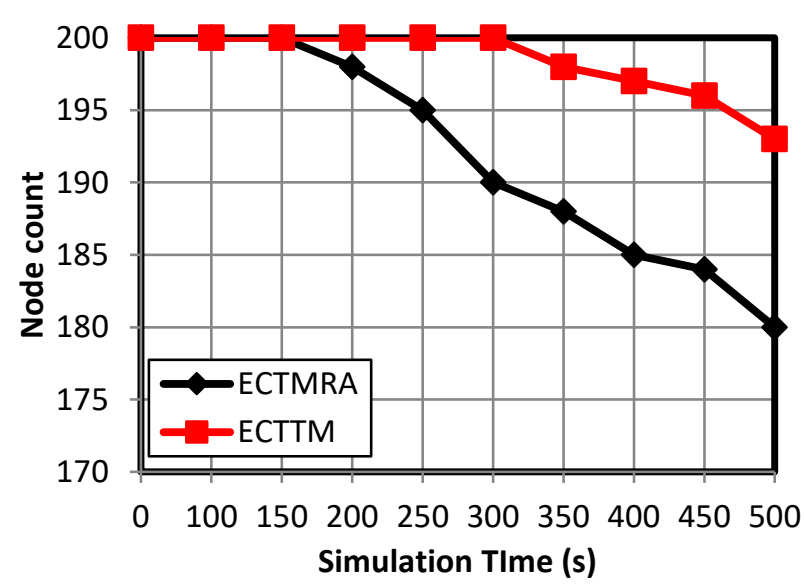

Figure.4 Network lifetime analysis

consumes more energy for computing trust score of both nodes and path. Additionally, the established routes are ranked one after the other and the first ranking route is selected for routing. All these processes consume more energy, which in turn decreases the network lifetime. Conversely, ECTTM maximizes the lifetime of the network, as it involves zero overhead in node clustering activity. The nodes are clustered easily, as the grid based structure is utilized. Additionally, the functioning nodes are limited, which reduces the energy consumption and thereby increasing the network lifetime.

The energy consumption analysis of ECTTM proves its energy efficiency, when compared to ECTMRA. ECTTM has attained this result because of controlling the count of functioning nodes per grid. Besides this, the clustering process of ECTTM does not involve any overhead, as the clusters are formed with respect to the geographical coordinates.

\subsection{Lifetime analysis}

Finally, the lifetime of the network with ECTTM and ECTMRA are compared. Both these algorithms are based on cluster and trust concept. However, ECTMRA does not have control over the state of the node. On the contrary, ECTTM controls the state of the node and allows only a limited count of nodes to be in functioning state. This enhances the network lifespan. The lifetime of the network is analysed with respect to the simulation time. The experimental results are presented below in Fig.4. Initially, two hundred nodes are deployed and the network lifetime analysis is performed. For the first 200 seconds, no node deaths occur. However, the event of node death is observed from $200^{\text {th }}$ second for ECTMRA with the death of two nodes. Again, three more nodes failed at the $250^{\text {th }}$ second on ECTMRA, whereas all the 200 nodes are alive on ECTTM. A big variation is observed in the $300^{\text {th }}$ second, in which all the nodes are alive on ECTTM and ten nodes failed in ECTMRA. At last, during $500^{\text {th }}$ second, 193 nodes are alive on ECTTM and 180 nodes are alive on ECTMRA.

On observation, it is evident that the proposed ECTTM is energy efficient because of the incorporation of cluster and trust concept. Besides this, the duty cycle of the sensor nodes are scheduled and controlled by the chief node. As the count of functioning nodes is limited, the lifetime of the network is considerably improved and thus, the objective of the work is attained.

\section{Conclusion}

This paper presents an energy efficient, cluster based and trustworthy Energy Conserving Trustworthy Topology Management (ECTTM) algorithm. ECTTM is proved to be energy efficient, owing to the presence of effective cluster formation and trust concept. The network area is divided into equal sized grids and the nodes are deployed in all the grids. The cluster is formed with respect to the location coordinates and the chief node is selected. The main role of the chief node is to compute the trust degree and to manage the state of the nodes. The entire process doesn't consume much energy and neither memory nor communication overhead is involved in ECTTM. The reason for minimal energy consumption is the control of functioning nodes. As minimal count of nodes are in functioning state, the remaining nodes are set to sleep, which greatly contributes to network lifetime enhancement. The proposed work is analysed in terms of network lifetime by varying the count of functioning nodes and the total number of nodes in the network. The experimental results show the efficacy of the proposed work. In future, a security pattern is planned to be introduced into the work. Additionally, 
the count of functional nodes is planned to be chosen by a bio-inspired algorithm.

\section{References}

[1] T. Rault, A. Bouabdallah, and Y. Challal, "Energy Efficiency in Wireless Sensor Networks: a top-down survey", Computer Networks, Vol.67, No. 4, pp. 104-122, 2014.

[2] Y. Wang, "Topology control for wireless sensor networks", Wireless Sensor Networks and Applications, Chapter 5, pp. 113-147, 2008.

[3] P. Santi, "Topology control in wireless ad hoc and sensor networks," ACM Comput. Surveys, Vol. 37, No. 2, pp. 164-194, 2005.

[4] Z. Gengzhong and L. Qiumei, "A survey on topology control in wireless sensor networks," In: Proc. of International IEEE Conference on Future Networks., Hainan, China, pp. 376-380, 2010.

[5] O. Younis, M. Krunz, and S. Ramasubramanian, "Node clustering in wireless sensor networks: Recent developments and deployment challenges," IEEE Networks, Vol. 20, No. 3, pp. 20-25, 2006.

[6] C. Chou, K. Ssu, H. Jiau, W. Wang, and C. Wang. "A dead-end free topology maintenance protocol for geographic forwarding in wireless sensor networks", IEEE Transactions on Computers, Vol. 60, No.11, pp.1610-1621, 2011.

[7] S. Özdemir, A.A. Bara'a, and ÖA. Khalil, "Multi-objective clustered-based routing with coverage control in wireless sensor networks", Soft Computing. Vol.17, No.9, pp.1573-1584, 2013.

[8] D. Z. Du and P. Pardalos, "Handbook of Combinatorial Optimization: Supplement", Vol.1, Springer Science and Business Media, 2013.

[9] W. Jie, G. Ming, and I. Stojmenovic, "On calculating power-aware connected dominating sets for efficient routing in ad hoc wireless networks", In: Proc. International Conference on Parallel Processing, Valencia, Spain, pp. $346-354,2001$.

[10] Z. Yuanyuan, X. Jia, and H. Yanxiang, "Energy efficient distributed connected dominating sets construction in wireless sensor networks", In: Proc. 2006 International Conference on Wireless Communications and Mobile Computing, British Columbia, Canada, pp.797802, 2006.

[11] M. A. Labrador and P. M. Wightman. "Topology Control in Wireless Sensor
Networks with a companion simulation tool for teaching and research", Springer Science and Business Media, 2009.

[12] L. Bao and J. J. Aceves, "Topology management in ad hoc networks", In: Proc. of ACM International Symposium on Mobile Ad Hoc Networking \& computing, New York, USA, pp.129-140, 2003.

[13] Z. Pawlak, "Rough sets, decision algorithms and Bayes' theorem", European Journal of Operational Research, Vol.136, No. 1, pp. 181189, 2002.

[14] T. Senthil and B. Kannapiran, "ECTMRA: Energy Conserving Trustworthy Multipath Routing Algorithm Based on Cuckoo Search Algorithm", Wireless Personal Communications, Vol.88, No.3, pp.1-20, 2016. 